Revista Thema

\begin{tabular}{l|l|l} 
v.20 Especial 2021
\end{tabular}

p.303-314
DOI: http://dx.doi.org/10.15536/thema.V20.Especial.2021.303-314.1857

ISSN: 2177-2894 (online)

\title{
Levantamento sobre dificuldades que interferem na vida acadêmica de universitários durante a pandemia de COVID-19
}

\author{
Survey on difficulties that interfer in the academic life of \\ university students during the COVID-19 pandemic \\ Alessandra Blando ${ }^{1}$, Fabiane Cristina Pereira Marcilio², \\ Sérgio Roberto Kieling Franco ${ }^{3}$, Marco Antônio Pereira Teixeira ${ }^{4}$
}

\section{RESUMO}

Este artigo tem por objetivo apresentar os principais resultados de um levantamento realizado por um serviço de apoio ao estudante de uma universidade federal sobre dificuldades que interferem na vida acadêmica dos estudantes durante a pandemia de COVID-19. Para tal, foi utilizado um questionário online, sendo uma pergunta aberta e outra fechada sobre essa temática. As respostas foram submetidas à análise estatística descritiva. No total, responderam ao levantamento 1.637 alunos, sendo $64 \%$ da graduação e $36 \%$ da pós-graduação. As principais dificuldades mencionadas pelos estudantes estão relacionadas com a gestão do tempo, os estudos e à saúde mental, além de outras voltadas à pandemia: como cuidar de sua saúde mental, realizar atividades físicas e manter os hábitos alimentares. Também foram apresentadas dificuldades de pensar o futuro profissional diante de um cenário de incertezas. Os dados sugerem que a maior parte dessas situações e dificuldades já faziam parte do contexto universitário, contudo parecem ter se agravado durante o período da pandemia, portanto, as instituições de educação superior necessitam incrementar suas ações de apoio à permanência dos estudantes.

Palavras-chave: Estudantes universitários; dificuldades acadêmicas; ensino superior; pandemia; COVID-19.

\section{ABSTRACT}

This article aims to present the main results of a survey carried out by a student support service at a federal university on difficulties that interfere in the academic life of students

\footnotetext{
${ }^{1}$ Doutoranda do Programa de Pós-Graduação em Educação, Universidade Federal do Rio Grande do Sul UFRGS, Porto Alegre/RS - Brasil. E-mail: alessandra.blando@ufrgs.br

2 Mestranda do Programa de Pós-Graduação em Psicologia e Saúde, Universidade Federal de Ciências da Saúde de Porto Alegre - UFCSPA, Porto Alegre/RS - Brasil. E-mail: fabiane_marcilio@hotmail.com

${ }^{3}$ Universidade Federal do Rio Grande do Sul - UFRGS, Porto Alegre/RS - Brasil. E-mail: sergio.franco@ $\underline{\text { ufrgs.br }}$

${ }^{4}$ Idem. E-mail: mapteixeira.psi@gmail.com
} 
during the COVID-19 pandemic. For this purpose, an online questionnaire was used, with one open question and one closed question on this topic. The answers were submitted to descriptive statistical analysis. In total, 1.637 students responded to the survey, $64 \%$ of them undergraduate and $36 \%$ postgraduate. The main difficulties mentioned by students are related to time management, studies and mental health, as well as others related to the pandemic: how to take care of your mental health, perform physical activities, and maintain eating habits. Difficulties were also presented in thinking about the professional future in the face of a scenario of uncertainty. The data suggest that most of these situations and difficulties were already part of the university context, however they seem to have worsened during the period of the pandemic, therefore, higher education institutions need to increase their actions to support the permanence of students.

Keywords: University students; academic difficulties; higher education; pandemic; COVID-19.

\section{INTRODUÇÃO}

Uma pandemia pode ser definida como a disseminação mundial de uma doença que afeta simultaneamente um grande número de pessoas. Em função de sua maior dimensão, seja pela sua propagação territorial ou pela sua gravidade de ocorrência, resulta em um número expressivo de casos graves ou óbitos. (VENTURA; SILVA, 2008). De acordo com a Organização Mundial da Saúde (OMS) ${ }^{5}$, em dezembro de 2019, iniciou-se na China um surto de COVID-19, uma doença causada pelo coronavírus SARS-CoV-2. Desde então, a doença começou a espalhar-se por outros países do mundo até chegar ao Brasil, tendo seu primeiro caso notificado em 20 de fevereiro de $2020^{6}$. Após, com o aumento do número de casos, o isolamento social foi adotado como uma medida prevenção de disseminação da COVID-19. Por conta disso, diversas empresas, escolas e universidades suspenderam suas atividades presenciais, reconfigurando novos espaços para trabalho e estudos. (ARRUDA, 2020; LOSEKANN; MOURÃO, 2020)

No que tange à educação superior no Brasil, a Portaria no 343 de 2020, emitida pela, Ministério da Educação, indicou a possibilidade da substituição das aulas presenciais por aula em meios digitais enquanto durasse a situação da pandemia de COVID-19. Com a suspensão das atividades presenciais das universidades e a adoção de trabalho remoto, diversos setores dessas instituições precisaram adaptar sua forma de atender os alunos. Diante disso, percebeu-se a importância de compreender como os estudantes universitários estão vivenciando esse momento. Nesse sentido, um serviço de apoio ao estudante de uma universidade federal da região sul do país, que tem como propósito contribuir para a construção de carreira e para o aprimoramento da aprendizagem, realizou um levantamento sobre as principais dificuldades que interferem na vida acadêmica dos alunos de graduação e de pós-graduação. Contudo, entende-se que antes de compreender como a pandemia de COVID-19 afeta a vida

\footnotetext{
${ }^{5}$ Traduzido e adaptado de https://www.who.int/emergencies/diseases/novel-coronavirus-2019. Acessado em 26 de junho de 2020.

${ }^{6}$ Informação obtidas através do site do Ministério da Saúde: https://covid.saude.gov.br. Acessado em 26 de junho de 2020.
} 
acadêmica de estudantes, é importante levar em conta as dificuldades que já fazem parte do contexto universitário em um período normal.

Os universitários enfrentam vários desafios ao longo da trajetória acadêmica, que não se resumem apenas a questões de estudos. Existem diferentes fatores que podem se apresentar como dificuldades no contexto do ensino superior. Estudos como os de Blando (2015) e Dias, Carlotto, Oliveira e Teixeira (2019) apresentam algumas das principais dificuldades vivenciadas pelos estudantes de graduação, tais como: decepção com as disciplinas e com o currículo do curso; descontentamentos com professores e a didática; desmotivação para estudar e frequentar aulas; dificuldade em gerir o tempo e lidar com a procrastinação; dificuldade em relacionar conteúdos e prática profissional; experiências insatisfatórias de estágio ou trabalho; entre outros.

Já na pós-graduação, há dificuldades específicas desse nível de ensino. Os estudantes de mestrado e doutorado necessitam, além de realizar sua pesquisa, escrever e ler artigos, gerir o tempo de estudos com sua vida particular. Os programas de pós graduação, visando manter ou aumentar sua excelência, acabam exigindo constantemente dos alunos um aumento no volume de publicações acadêmicas, o que leva a uma cultura de produtivismo que tende a sobrecarregar os pesquisadores e gerar problemas relacionados à saúde mental. Ainda, estudos indicam outras dificuldades percebidas pelos mestrandos e doutorandos, tais como falta de motivação para realizar as pesquisas, relacionamento distante com orientadores e/ou colegas, incertezas quanto ao futuro profissional, entre outras. (FARO, 2013; CESAR, SOUSA, RIBEIRO; OLIVEIRA, 2018)

Nesse sentido, essas dificuldades referidas anteriormente podem afetar a saúde mental dos estudantes da graduação e da pós-graduação de maneira significativa e preocupante. Estudos demonstram que entre estudantes de graduação e, especialmente, nos de pós-graduação, as taxas de ansiedade, depressão, crise de pânico, distúrbios do sono, além do risco de suicídio são bem maiores quando comparadas à população em geral. (COSTA; NEBEL, 2018; PADOVANI et al., 2014).

Identificar as dificuldades vivenciadas pelos estudantes no presente momento pode contribuir para o desenvolvimento de ações a serem desenvolvidas no período da pandemia. Diante disso, o presente trabalho teve como objetivo apresentar os principais resultados do levantamento realizado pelo serviço de apoio ao estudante de uma universidade federal brasileira acerca das principais dificuldades que interferem na vida acadêmica dos estudantes durante o período de distanciamento social causado pela pandemia de COVID-19.

\section{MÉTODO}

Trata-se de um estudo quantitativo, a partir de dados retirados de um questionário enviado aos estudantes da Universidade. O serviço de apoio universitário realizou, entre os dias 27 de abril de 2020 e 04 de maio de 2020, um levantamento junto aos estudantes sobre necessidades e dificuldades vivenciadas durante o período de 
distanciamento social. O instrumento utilizado foi um breve questionário online e anônimo divulgado aos alunos através das mídias sociais do serviço e de e-mails encaminhados pelas secretarias dos cursos de graduação e de pós-graduação. 0 questionário apresentava oito perguntas (cinco fechadas e três abertas) com o propósito de verificar o vínculo do aluno com a universidade (graduação ou pósgraduação), identificar as principais dificuldades vivenciadas nesse momento de isolamento social, verificar as plataformas e formatos online mais utilizados para acessar conteúdos e informações e solicitar sugestões de possíveis atividades a serem oferecidas pelo serviço durante o período de isolamento social. Para o presente estudo, foram analisadas as respostas de apenas duas perguntas: 1) Quais suas três maiores dificuldades durante esse período de quarentena? (questão fechada) e 2) Quais suas dificuldades acerca da vida acadêmica no momento presente? (questão opcional e aberta). As respostas de ambas as perguntas foram submetidas à análise estatística descritiva, sendo que na pergunta aberta primeiramente realizou-se uma leitura prévia e, após, as respostas foram agrupadas de acordo com oito categorias criadas e analisadas em termos de frequência. Salienta-se que o levantamento não teve como propósito identificar o perfil da amostra, com exceção do vínculo do aluno com a universidade (graduação ou pós-graduação).

\section{RESULTADOS}

Responderam ao levantamento um total de 1.637 alunos, sendo 64\% (1.048) da graduação e 36\% (589) da pós-graduação. A Tabela 1 apresenta as respostas da pergunta: "Quais suas três maiores dificuldades durante esse período de quarentena?" Essa questão era fechada com a possibilidade de assinalar mais de uma opção de resposta, incluindo uma lista de nove dificuldades mais a alternativa "outros" (para que o aluno pudesse escrever livremente).

Tabela 1 - Frequência e porcentagens das dificuldades dos estudantes durante a pandemia.

\begin{tabular}{llll}
\hline $\begin{array}{l}\text { Dificuldades durante a } \\
\text { pandemia }\end{array}$ & $\begin{array}{l}\text { Total } \\
\text { (n= 1637) }\end{array}$ & $\begin{array}{l}\text { Graduação } \\
\text { (n= 1048) }\end{array}$ & $\begin{array}{l}\text { Pós-graduação } \\
\text { (n= 589) }\end{array}$ \\
\hline Lidar com a procrastinação & $55,7 \%(911)$ & $56,6 \%(593)$ & $54 \%(318)$ \\
Estabelecer uma rotina & $54 \%(885)$ & $57,4 \%(602)$ & $48 \%(283)$ \\
Fazer atividades físicas & $45,8 \%(750)$ & $47,6 \%(499)$ & $42,6 \%(251)$ \\
Estudar & $38,5 \%(631)$ & $46,9 \%(491)$ & $23,9 \%(140)$ \\
Cuidar da saúde mental & $33 \%(541)$ & $33,4 \%(351)$ & $32,2 \%(190)$ \\
Manter hábitos alimentares & $26,6 \%(435)$ & $30,6 \%(321)$ & $19,4 \%(114)$ \\
Escrever TCC, Dissertação ou Tese & $26,4 \%(433)$ & $12,5 \%(131)$ & $51,3 \%(302)$ \\
Pensar no futuro profissional & $24,7 \%(404)$ & $23,3 \%(244)$ & $27,2 \%(160)$ \\
Manter contato com & $17,3 \%(284)$ & $18,2 \%(190)$ & $15,9 \%(94)$ \\
amigos/familiares & $3 \%(50)$ & $2,3 \%(24)$ & $4,4 \%(26)$ \\
Outros & &
\end{tabular}

Fonte: Elaborada pelos autores. 
No total, as dificuldades mais assinaladas pelos estudantes foram: Lidar com a procrastinação $(55,7 \%)$, Estabelecer uma rotina $(54 \%)$, Fazer exercícios físicos (45,8\%), Estudar (38,5\%) e Cuidar da saúde mental (33\%). Pode-se verificar diferenças na ordem de posição das dificuldades assinalada entre os estudantes de graduação e pós-graduação. Considerando apenas os alunos da graduação, as quatro mais apontadas foram: 1o Estabelecer uma rotina (57,4\%), 2ㅇ Lidar com a procrastinação (56,6\%), 3o Fazer atividades físicas (47,6\%) e 4ㅇ Estudar (46,9\%). Já entre os alunos da pós-graduação, as quatro mais assinaladas foram: 1 Lidar com a procrastinação (54\%), 2o Escrever Dissertação ou Tese (51,3\%). 3을 Estabelecer uma rotina (48\%) e 4응 Fazer atividades físicas (42,6\%). Quanto ao percentual, três dificuldades apresentaram valores mais distintos entre os dois grupos de respondentes: Escrever TCC, Dissertação ou Tese (12,5\% graduação e 51,3\% pós-graduação ${ }^{7}$ ); Manter hábitos alimentares (30,6\% graduação e 19,4\% pós-graduação); e Estudar (46,9\% graduação e $23,9 \%$ pós-graduação).

As respostas à pergunta aberta "Quais suas dificuldades acerca da vida acadêmica no momento presente?" foram primeiramente categorizadas e depois analisadas em termos de frequência, conforme apresentado na Tabela 2 . Um total de 988 (60,3\% da amostra) responderam essa questão, sendo $604(61,1 \%)$ alunos da graduação e 385 $(38,9 \%)$ da pós-graduação.

Tabela 2 - Categorias das dificuldades acerca da vida acadêmica durante a pandemia.

\begin{tabular}{|c|c|c|c|}
\hline Categorias & Descrição & Exemplos de respostas & $\begin{array}{l}\text { Total } \\
(n=988)\end{array}$ \\
\hline Estudos & $\begin{array}{l}\text { Falta de motivação, não } \\
\text { conseguir se concentrar, falta de } \\
\text { rotina de estudos, dificuldades } \\
\text { para escrever e estudar em casa }\end{array}$ & $\begin{array}{l}\text { "Não ter vontade nenhuma } \\
\text { de estudar" }\end{array}$ & $32,2 \%(318)$ \\
\hline Saúde mental & $\begin{array}{l}\text { Ansiedade frente à pandemia, } \\
\text { desânimo, sentimentos de } \\
\text { incerteza e preocupação quanto } \\
\text { ao futuro }\end{array}$ & $\begin{array}{l}\text { "A maior dificuldade é a } \\
\text { ansiedade de não saber } \\
\text { quando tudo vai voltar ao seu } \\
\text { normal" }\end{array}$ & $16,9 \%(167)$ \\
\hline Gestão do tempo & $\begin{array}{l}\text { Administração do tempo, } \\
\text { conciliar diferentes tarefas, ter } \\
\text { uma rotina, lidar com a } \\
\text { procrastinação }\end{array}$ & $\begin{array}{l}\text { "Dificuldade de ter uma } \\
\text { rotina com tempo de } \\
\text { estudos" }\end{array}$ & $14,6 \%(144)$ \\
\hline $\begin{array}{l}\text { Suspensão das } \\
\text { atividades } \\
\text { presenciais }\end{array}$ & $\begin{array}{l}\text { Impossibilidade de acesso aos } \\
\text { laboratórios e bibliotecas, falta } \\
\text { das aulas presenciais e interação } \\
\text { com colegas e professores }\end{array}$ & $\begin{array}{l}\text { "As relações com colegas e } \\
\text { professores são inexistentes } \\
\text { durante a quarentena" }\end{array}$ & $12,3 \%(121)$ \\
\hline
\end{tabular}

\footnotetext{
${ }^{7}$ Sabe-se que todos alunos de pós-graduação escrevem tese ou dissertação, mas somente uma pequena parcela dos de graduação estão dedicados ao trabalho de conclusão de curso (TCC), tanto por este ocorrer no final do curso como também porque há cursos que não o exigem, o que explica um maior número entre estudantes de pós-graduação.
} 


\begin{tabular}{|c|c|c|c|}
\hline Carreira & $\begin{array}{l}\text { Planejamento de carreira, } \\
\text { dúvidas sobre a escolha do } \\
\text { curso, incerteza quanto ao } \\
\text { futuro profissional e inserção no } \\
\text { mercado de trabalho. }\end{array}$ & $\begin{array}{l}\text { "Incertezas quanto à vida } \\
\text { profissional pós coronavírus" }\end{array}$ & $11,4 \%(113)$ \\
\hline $\begin{array}{l}\text { Falta de } \\
\text { assistência da } \\
\text { universidade }\end{array}$ & $\begin{array}{l}\text { Falta de comunicação com os } \\
\text { alunos, falta de informações } \\
\text { sobre calendário acadêmico e } \\
\text { aulas EaD. }\end{array}$ & $\begin{array}{l}\text { "O sentimento de abandono } \\
\text { por parte dos professores e } \\
\text { coordenação do curso" }\end{array}$ & $6,2 \%(61)$ \\
\hline Não se aplica & Não relatou dificuldades. & $\begin{array}{l}\text { "Nenhuma no momento, } \\
\text { apenas saudades e falta da } \\
\text { vida normal de aulas" }\end{array}$ & $3,5 \% \%(35)$ \\
\hline $\begin{array}{l}\text { Dificuldades } \\
\text { econômicas }\end{array}$ & $\begin{array}{l}\text { Falta de acesso à internet e } \\
\text { recursos tecnológicos, corte de } \\
\text { bolsas, dificuldades financeiras, } \\
\text { não conseguir estágio/ trabalho. }\end{array}$ & $\begin{array}{l}\text { "A dificuldade em usar } \\
\text { internet, pois não tenho } \\
\text { condições de adquirir } \\
\text { assinatura Wi-Fi" }\end{array}$ & $2,9 \%(29)$ \\
\hline
\end{tabular}

Fonte: Elaborada pelos autores.

As dificuldades acadêmicas descritas pelos alunos se agruparam principalmente na categoria Estudos (32,2\%), Saúde Mental (16,9\%) e Gestão do Tempo (14,6\%). Ao analisar em separado as respostas entre os dois grupos (alunos de graduação e de pós-graduação) verificou-se que os percentuais em cada categoria foram semelhantes. Assim, optou-se apresentar apenas os resultado gerais de todos os respondentes.

\section{DISCUSSÕES}

Diante dos resultados apresentados, de modo geral, torna-se evidente que as principais dificuldades apresentadas pelos estudantes universitários durante a pandemia, em que as aulas estavam suspensas devido à necessidade de isolamento social, estão relacionadas aos estudos, gestão do tempo e saúde mental. Tais dificuldades, antes da ocorrência da pandemia, já faziam parte do contexto universitário, entretanto, parecem ter se agravado durante o isolamento social. Além disso, fatores associados ao autocuidado - cuidar de si, de sua saúde e do seu bemestar - realizar atividade físicas e manter hábitos alimentares foram considerados. Possivelmente, essas dificuldades estejam mais diretamente ligadas ao contexto da pandemia e não necessariamente apareceriam em um momento considerado normal. Além disso, outra dificuldade significativa está relacionada à carreira, especialmente no que diz respeito a pensar o futuro. Incertezas quanto ao mercado de trabalho e à crise econômica durante e pós pandemia podem colaborar com essa dificuldade. Ressalta-se ainda que apenas uma pequena parte dos alunos respondeu que não percebiam dificuldades acadêmicas vivenciadas durante a pandemia. Entretanto, é preciso considerar que o levantamento foi realizado em um período de suspensão de aulas presenciais, e a universidade, até aquele momento, não tinha ainda estruturado um plano de retorno às atividades, mesmo que de maneira remota. 
Dificuldades relacionadas à organização do tempo foi uma das mais assinaladas pelos respondentes. O contexto universitário demanda do estudante um bom gerenciamento do tempo para conseguir conciliar as atividades da vida pessoal com as demandas acadêmicas, que incluem cargas de leituras, escrita e entrega de trabalhos nos prazos, além do estabelecimento de uma rotina de estudos. A frequência de problemas de administração do tempo entre os universitários, mesmo antes do período da pandemia do COVID-19, é bastante elevada (DIAS et al., 2019), destacando-se principalmente comportamentos de procrastinação de atividades acadêmicas. Estudos indicam que a incidência estimada desse fenômeno entre os estudantes é de 80 a 95\%, o que evidencia sua predominância no ensino superior. (SAMPAIO; BARIANI, 2011; STEEL, 2007). No levantamento, lidar com a procrastinação foi a dificuldade mais assinalada entre os respondentes.

Os problemas para gerenciar o tempo, estabelecer uma rotina e de saber que atividades priorizar no cotidiano podem ser obstáculos ao autocuidado, a partir do que foi mencionado pelos estudantes, que indicaram dificuldades para conseguir fazer atividades físicas e manter os hábitos alimentares durante a pandemia. $\mathrm{O}$ isolamento social pode restringir os tipos de atividades físicas a serem realizadas, com o fechamento de academias, centros de treinamento, clubes etc. Ainda, compras online de alimentos e bebidas para consumo imediato, de acordo com o estudo "Novos hábitos digitais em tempos de COVID-19", realizado pela Sociedade Brasileira de Varejo e Consumo (SBVC, 2020), apresentou um aumento de mais de $79 \%$. Isso pode indicar que por conta de uma má gestão do tempo ou da necessidade de conciliar diversas demandas (trabalho em casa, convívio familiar, atividades domésticas, estudos etc.) dificuldades para manter os hábitos alimentares podem surgir ou se acentuarem, quando comparadas a um período pré-pandemia.

Até o momento, não foram encontrados estudos publicados sobre a gestão do tempo de universitários durante a pandemia. No entanto, a partir dos relatos das respostas da pergunta aberta, percebeu-se que o contexto de pandemia, e a necessidade de isolamento social, pode ter aumentado o volume de tarefas, como o de atividades domésticas por se passar mais tempo em casa. Essas tarefas aliadas ao convívio familiar (cuidar dos filhos, de pessoas que são grupo de risco, ter de dividir espaços para trabalho e/ou estudos, realizar vídeo chamadas para entrar em contato com amigos e familiares etc.) e aos procedimentos de cuidado para evitar a contaminação por coronavírus (higienização de compras do supermercado, de sapatos, roupas, uso de máscaras etc.) podem dificultar o estabelecimento de prioridades e o manejo do tempo no dia a dia. As pessoas estão tendo de aprender a dividir, em um mesmo ambiente, atividades de trabalho, acadêmicas, domésticas e de lazer. Ademais, em geral, elas acabam não percebendo o tempo que ficam envolvidas nas diferentes tarefas, o que pode levar a uma percepção equivocada do verdadeiro uso do tempo. (LOSEKANN; MOURÃO, 2020). Diante disso, estudantes podem se sentir improdutivos por não estarem estudando ou escrevendo seus trabalhos acadêmicos (trabalhos de conclusão de curso, dissertação ou tese). Essa sensação de improdutividade pode gerar sentimentos de culpa, prejudicando, por sua vez, sua saúde mental. 
Dificuldades relativas à saúde mental de estudantes, tanto de graduandos, quanto de pós-graduandos, já fazem parte do contexto universitário em um período normal. (COSTA; NEBEL, 2018; PADOVANI et al., 2014). Um estudo recente de Maia e Dias (2020), sugere que há um aumento significativo de problemas psicológicos ansiedade, depressão e estresse - entre universitários durante a pandemia se comparado a períodos normais. Os resultados obtidos por Maia e Dias (2020) estão alinhados a estudos internacionais que analisaram o efeito psicológico da COVID-19 e de outras pandemias. (WANG et al., 2020; WEISS; MURDOCH, 2020). Se considerarmos uma análise global, das respostas da pergunta Quais suas três maiores dificuldades durante esse período de quarentena?, mais de $30 \%$ dos estudantes revelaram que estavam com dificuldades de cuidar de sua saúde mental, o que é corroborado pelas respostas da questão aberta, que mencionam, especialmente, ansiedade e angústia de lidarem com esse momento, seja relacionado a fatores acadêmicos ou pela situação da pandemia em si. Desse modo, tais resultados sugerem que a pandemia pode prejudicar a saúde psíquica dos estudantes, inclusive agravando situações preexistentes.

Além disso, entende-se que dificuldades mencionadas anteriormente sobre gestão do tempo e de saúde mental podem relacionar-se com a falta de motivação para estudar durante esse período de pandemia. Também é importante considerar o impacto das notícias acerca do número de infectados e mortos no país. A suspensão de aulas presenciais, a falta de interação com colegas e professores e questões voltadas ao autogerenciamento de seus estudos, exigirão maior autonomia do estudante diante de sua vida acadêmica, uma vez que os cursos presenciais utilizam uma série de medidas e rituais que, ainda que não sejam suficientes, ajudam o estudante a se organizar.

Ainda, no momento em que os estudantes responderam ao levantamento, a universidade não havia informado sobre como seria um possível retorno às aulas, nem mesmo de modo online, o que gerou nos alunos sentimentos de angústia diante de um cenário de incerteza sobre sua vida acadêmica. Alguns estudantes também apresentaram preocupação quanto ao acesso à internet no caso da implementação da modalidade de ensino remoto. Dados da $\mathrm{V}$ Pesquisa Nacional de Perfil Socioeconômico e Cultural dos Graduandos das Instituições Federais de Ensino Superior (ANDIFES, 2018) indicam que cerca de $10 \%$ das famílias brasileiras de estudantes de graduação não têm acesso à internet e mais de $12 \%$ não possuem acesso a computadores (isso sem levar em conta a posse de celulares). Apesar desses percentuais não serem elevados, é preciso considerar que o atual contexto de pandemia e isolamento social irão impactar significativamente a situação econômica do país, afetando diretamente as condições financeiras da população e, consequentemente, a capacidade de manter e, até mesmo, de adquirir acesso à internet. (ARRUDA, 2020). Nos resultados obtidos neste estudo, o número de estudantes que relataram essa preocupação não foi significativo se comparado a outras dificuldades mencionadas, entretanto, é preciso considerar que provavelmente quem tem menos acesso à internet pode não ter respondido ao levantamento. 
Por fim, uma parte dos alunos referiram dificuldades voltadas à carreira, como a incerteza em relação ao futuro junto ao medo de não conseguir emprego ou estágio em função da instabilidade do mercado de trabalho por conta da crise econômica provocada pela pandemia. Preocupações sobre o futuro e a inserção profissional após a formação acadêmica já eram um problema enfrentado pelos estudantes tanto de nível de graduação (TEIXEIRA; GOMES, 2004; VALORE; SELIG, 2010), como de pósgraduação. (CESAR et al., 2018; FARO, 2013). Antes mesmo da pandemia, as transformações e a competitividade do mundo do trabalho já afetavam de maneira significativa aqueles que estavam buscando emprego, e o diploma de curso superior, há algum tempo, deixou de ser uma garantia de uma boa colocação e remuneração no mercado profissional. (TEIXEIRA; GOMES, 2004). Some-se a isso a possibilidade de adiamento das colações de grau, em função da suspensão de atividades letivas. Todavia, as incertezas sobre a economia global geradas pela pandemia parecem ter intensificado essas preocupações.

\section{CONSIDERAÇÕES FINAIS}

A partir desse levantamento, foi possível compreender as principais necessidades dos estudantes durante esse período de pandemia da COVID-19, destacando-se as dificuldades relacionadas à gestão do tempo, à organização da rotina, à procrastinação, aos estudos, ao envolvimento em atividades físicas e à escrita de dissertação, tese ou TCC. Os dados indicam ainda que cerca de 30\% dos estudantes manifestaram preocupação com sua saúde mental neste momento. A saliência do tema da saúde mental entre universitários não é novidade (COSTA; NEBEL, 2018; PADOVANI et al., 2014), sendo uma questão que já estava presente no contexto universitário antes da pandemia e da suspensão das atividades acadêmicas presenciais.

Além disso, nesse momento os alunos parecem sentir uma maior necessidade de escuta de suas dificuldades. Ainda que muitas respostas sejam difíceis de serem dadas pela instituição na situação de pandemia, a possibilidade de poderem expressar suas preocupações pode mitigar parte das ansiedades que as atuais circunstâncias produzem, contribuindo para a redução do sofrimento mental. Ademais, a percepção de que existe um interesse institucional pelos alunos pode auxiliar na manutenção da motivação em relação aos estudos e à própria universidade, sendo um fator potencialmente preventivo à evasão quando houver a retomada das atividades acadêmicas, seja de maneira remota ou presencial.

Deste modo, acredita-se que seria importante estimular que as universidades criem canais de comunicação efetivos com seus alunos para abordar dúvidas e questões referentes à vida acadêmica durante a suspensão das aulas e o futuro. Mais do que apenas disponibilizar e-mail para comunicação, é importante a criação de espaços para encontros virtuais nos quais alunos possam interagir sincronamente com alguém que possa ouvir e dar algum encaminhamento às demandas que surgirem. A criação de canais virtuais de acolhimento e orientação para dificuldades de caráter mais 
psicológico, psicopedagógico ou de assistência social é também uma alternativa. Esses canais de comunicação poderiam ser centralizados e serviriam também para dar ao estudante a percepção de suporte institucional.

Ainda, entende-se que, na iminência de um retorno às aulas, mesmo que na modalidade remota, para além de oferecer capacitação adequada aos docentes e técnicos das universidades, é preciso levar em conta as dificuldades relatadas pelos estudantes, principalmente, as relacionadas a questões econômicas, ao gerenciamento do tempo, à falta de motivação para estudar e à saúde mental. Sendo assim, deve-se atentar também à adaptação dos estudantes a essa nova modalidade enquanto as aulas não podem ser retomadas, tanto em questões técnicas de informática quanto na sua autogestão de estudos, pois eram oriundos de cursos presenciais. Ademais, é necessário criar formas de auxílio aos estudantes que não têm condições financeiras de aderirem a um ensino remoto para que não sejam prejudicados em sua vida acadêmica, evitando gerar uma exclusão social dentro da própria instituição.

Por fim, cabe salientar que muitas das necessidades dos estudantes observadas no levantamento já existiam previamente à suspensão das atividades acadêmicas, pois são características do contexto universitário. Os resultados obtidos no levantamento, entretanto, sinalizam que essas situações e dificuldades preexistentes poderão se agravar durante e após a pandemia. Isso aponta para a possibilidade de que, no retorno às atividades remotas ou presenciais, as demandas dos estudantes por apoio a questões de aprendizagem, saúde mental, carreira e de adaptação à universidade sejam ainda mais pronunciadas, o que precisa ser considerado nas instituições de educação superior para que se possa apoiar a permanência dos estudantes nas universidades.

\section{REFERÊNCIAS}

ANDIFES. V Pesquisa Nacional de Perfil Socioeconômico e Cultural dos (as)

Graduandos (as) das IFES. Associação Nacional dos Dirigentes das Instituições Federais de Ensino Superior, 2018. Disponível em: http://www.andifes.org.br/vpesquisa-nacional-de-perfil-socioeconomico-e-cultural-dos-as-graduandos-as-das-ifes2018/. Acesso em: 11 mai. 2020.

ARRUDA, E. P. Educação Remota Emergencial: elementos para políticas públicas na educação brasileira em tempos de COVID-19. EmRede - Revista de Educação à Distância, v.7, n.1, p.257-275, 2020

BRASIL. Portaria no 343, de 17 de março de 2020. Dispõe sobre a substituição das aulas presenciais por aulas em meios digitais enquanto durar a situação de pandemia do Novo Coronavírus - COVID-19. Brasília, DF: Ministério da Educação, 2020. Disponível em: http://www.planalto.gov.br/CCIVIL_03/Portaria/PRT/Portaria\%20n\%C2\%BA\%2034320-mec.htm. Acesso em: 26 jun. 2020. 
CESAR, F. C. R.; SOUSA, E. T.; RIBEIRO, L. C. M; OLIVEIRA, L. M. A. C. Estressores da pós-graduação: revisão integrativa da literatura. Cogitare enfermagem, Curitiba, v.23, n.4, e57460, 2018.

COSTA, E. G.; NEBEL, L. O quanto vale a dor? Estudo sobre a saúde mental de estudantes de pós-graduação no Brasil. Polis, Santiago, v.17, n.50, p.207-227, ago. 2018.

DIAS, A. C. G.; CARLOTTO, R. C.; OLIVEIRA, C.T.; TEIXEIRA, M. A. P. Dificuldades percebidas na transição para a universidade. Revista Brasileira de Orientação Profissional, São Paulo, v.20, n.1, p.19-30, jun. 2019.

FARO, André. Estresse e estressores na pós-graduação: estudo com mestrandos e doutorandos no Brasil. Psicologia: Teoria e Pesquisa, Brasília, v.29, n.1, p.51-60, mar. 2013.

LOSEKANN, R. G. C. B.; MOURÃO, H. C. Desafios do teletrabalho na pandemia COVID19: quando o home vira office. Caderno de Administração, v.28, p.71-75, 2020.

MAIA, B. R.; DIAS, P. C. Ansiedade, depressão e estresse em estudantes universitários: o impacto da COVID-19. Estudos de Psicologia, Campinas, v.37, p.18, 2020.

PADOVANI, R. C. et al. Vulnerabilidade e bem-estar psicológicos do estudante universitário. Revista Brasileira de Terapias Cognitivas, Rio de Janeiro, v.10, n.1, p.2-10, jun. 2014.

SAMPAIO, R. K. N.; BARIANI, I. C. D. Procrastinação acadêmica: um estudo exploratório. Estudos Interdisciplinares em Psicologia, Londrina, v.2, n.2, p.242-262, jun. 2011.

SBVC. Novos hábitos digitais em tempos de COVID-19. Sociedade Brasileira de Varejo e Consumo, 2020. Disponível em: http://sbvc.com.br/estudo-novos-habitosdigitais-em-tempos-de-covid-19/. Acesso em: 25 mai. 2020.

STEEL, P. The nature of procrastination: a meta-analytic and theoretical review of quintessential self-regulatory failure. Psychological Bulletin, v.133, n.1, p.65-94, 2007.

TEIXEIRA, M. A. P.; GOMES, W. B. Estou me formando... e agora?: Reflexões e perspectivas de jovens formandos universitários. Revista Brasileira de Orientação Profissional, São Paulo, v.5, n.1, p.47-62, jun. 2004.

VALORE, L. A.; SELIG, G. A. Inserção profissional de recém-graduados em tempos de inseguranças e incertezas. Estudos e Pesquisas em Psicologia, v. 10, p. 390-404, 2010.

VENTURA, D.; SILVA, M. La société internationale et les grandes pandémies. Resenha. Revista de Direito Sanitário, v.9, p.280-283, 2008. 
WANG, C.; PAN, R.; WAN, X.; TAN, Y.; XU, L.; HO, C. Immediate psychological responses and associated factors during the initial stage of the 2019 Coronavirus Disease (COVID-19) epidemic among the general population in China. International Journal of Environmental Research and Public Health, v.17, n.5, 2020.

WEISS, P.; MURDOCH, D. R. Clinical course and mortality risk of severe COVID-19. The Lancet, v.395, p.1014-1015, mar. 2020. 